\title{
A CONJECTURE OF R. NEVANLINNA CONCERNING THE GENUS OF A MEROMORPHIC FUNCTION
}

\author{
ALBERT EDREI ${ }^{1}$ AND S. M. SHAH ${ }^{2}$
}

1. Introduction. Let $f(z)$ be a meromorphic function of finite order $\lambda$ and genus $q$. We write $g(f)=q$, and, in general, denote by $g(h)$ the genus of $h(z)$.

It is well known that the order is invariant under differentiation and also that, if $\lambda$ is not a positive integer,

$$
g(f)=[\lambda] .
$$

Hence in this case,

$$
g\left(f^{\prime}\right)=g(f) .
$$

For positive integral values of $\lambda$, it is only possible to prove [ 1 , p. 104]

$$
g\left(f^{\prime}\right) \leqq g(f)
$$

and there exist functions for which the strict inequality holds.

In [1], R. Nevanlinna examines this inequality and its possible connections with the important notion of deficiency. Let $\delta(a, f)$ denote the deficiency of the value $a$, with respect to $f$, and let $\Delta(f)$ be the total deficiency of $f(z)$ :

$$
\Delta(f)=\sum_{a} \delta(a, f) .
$$

Nevanlinna [1, p. 106] conjectured that

$$
g\left(f^{\prime}\right)<g(f)
$$

implies

$$
\Delta(f)=2 .
$$

In this note we disprove this conjecture by constructing an entire function $f(z)$ of order one, for which (1) holds as well as

Presented to the Society June 20, 1959 under the title $A$ conjecture of Nevanlinna on meromorphic functions; received by the editors April 24, 1959 and, in revised form, May 8, 1959.

1 The research of this author was supported in part by a grant from the National Science Foundation.

${ }^{2}$ Sponsored by the United States Army under Contract No. DA-11-022-ORD2059. 


$$
\sum_{a \neq \infty} \delta(a, f)=0 .
$$

If $m(>1)$ is an integer, we may consider $f\left(z^{m}\right)$ and thus obtain functions of order $m$ for which (1) and (2) hold simultaneously.

2. Some properties of canonical products of genus zero with negative zeros. If all the zeros of a canonical product $h(z)$ are negative, it is clear that $h(z)$ is completely determined by the counting function $n(t)$ of its zeros. It is well known that $h(z)$ is of genus zero if and only if

$$
\int^{\infty} \frac{n(t) d t}{t^{2}}<\infty
$$

Then

$$
\log M(r, h)=\log h(r)=r \int_{0}^{\infty} \frac{n(t) d t}{t(t+r)}, \quad(r \geqq 0, n(0)=0) .
$$

An obvious integration yields

$$
\int_{1}^{\infty} r^{-2} \log M(r, h) d r=\int_{0}^{\infty} \frac{n(t)}{t^{2}} \log (1+t) d t,
$$

but it is important to observe that the convergence of (3) does not imply the convergence of the integrals in (5).

A simple example of this behavior is given by

$$
n(t) \sim t /(\log t)^{2}
$$$$
(t \rightarrow \infty) \text {. }
$$

Our construction depends on this observation and also on the possibility of introducing "gaps" in the sequence of zeros of $h(z)$, without inducing convergence of the integrals in (5).

Our gaps will be such that, for a suitable unbounded sequence of values of $R$,

$$
n(t) \equiv n\left(R^{1 / 3}\right)
$$

in the interval $\left(R^{1 / 3}, e^{R}\right)$.

By (4) the presence of such a gap implies

$$
\log M(R, h) \leqq O\left(n\left(R^{1 / 3}\right) \log R\right)+R \int_{e^{R}}^{\infty} \frac{n(t) d t}{t^{2}},(R \rightarrow \infty) .
$$

3. Construction and properties of a special entire function. Consider a positive increasing sequence 


$$
R_{1}, R_{2}, \cdots
$$

such that

$$
e^{R_{m}}<R_{m+1}^{1 / 3}
$$$$
m=1,2,3, \cdots .
$$

We take $R_{1}$ so large that $t /(\log t)^{2}$ increases for $t \geqq \exp \left(R_{1}\right)$ and define

$$
\begin{array}{lr}
n(t)=0 & \text { for } t<e^{R_{1}}, \\
n(t)=\left[\frac{t}{(\log t)^{2}}\right] & \text { for } e^{R_{m}} \leqq t \leqq R_{m+1}^{1 / 3}, m=1,2,3, \cdots, \\
n(t)=n\left(R_{m+1}^{1 / 3}\right) & \text { for } R_{m+1}^{1 / 3}<t<e^{R_{m+1}} .
\end{array}
$$

The condition (8) sets no upper bound on the ratio $R_{m+1} / R_{m}$ and since

$$
\int_{e^{R_{m}}}^{\infty}\left(\frac{t}{(\log t)^{2}}-1\right) \frac{\log t}{t^{2}} d t=\infty,
$$

it is clear that the members of (7) may be spaced so as to produce divergence of the integrals in (5). We assume that (7) has been selected in this manner. Then (9) determines uniquely $n(t)$ and hence a canonical product $h(z)$, of genus zero, for which

$$
\int_{1}^{\infty} r^{-2} \log M(r, h) d r=\infty .
$$

Moreover in view of (6), it is easily seen that the lower order of $h(z)$ does not exceed $1 / 3$.

4. Construction of a function satisfying (1) and (2). Since $h(z)$ is a canonical product of genus zero with negative zeros,

$$
\min _{|z|=r}|h(z)|=|h(-r)|, \quad r>0 .
$$

We have also shown that the lower order of $h(z)$ is less than $1 / 2$ so that by the theorem of Phragmén-Lindelöf, $|h(-r)|$ is unbounded and hence

$$
\delta(0, h)=0 .
$$

Consider some primitive $f_{0}(z)$ of $h(z)$ and put

$$
f_{1}(z)=f_{0}(z)+1
$$

so that 


$$
f_{0}^{\prime}(z)=f_{1}^{\prime}(z)=h(z) .
$$

If

$$
g\left(f_{0}\right)=g\left(f_{1}\right)=0,
$$

a theorem of Valiron [2, pp. 183-184] would imply

$$
\int^{\infty} r^{-2} \log M\left(r, f_{0}\right) d r<\infty .
$$

On the other hand, since $f_{0}(z)$ is of finite order [2, p. 36]

$$
\log M\left(r, f_{0}\right) \sim \log M\left(r, f_{0}^{\prime}\right)
$$

so that (12) and (13) contradict (10). Hence $\max \left(g\left(f_{0}\right), g\left(f_{1}\right)\right)=1$. Denote by $f(z)$ the first of the two functions $f_{0}, f_{1}$ which is of genus one. It clearly satisfies (1); it also satisfies (2) since this follows from (11) and (12) and the inequality [3, p. 22]

$$
\sum_{a \neq \infty} \delta(a, f) \leqq \delta\left(0, f^{\prime}\right) \text {. }
$$

5. Function of regular growth satisfying (1) and (2). The function $f(z)$ defined above is of irregular growth. We now construct another function $f(z)$ of regular growth order 1 satisfying (1) and (2). Consider the function

$$
h_{1}(z)=\prod_{n=2}^{\infty}\left\{1+\frac{z^{2}}{n^{2}(\log n)^{4}}\right\} .
$$

It is known that $[1, p .18]$

$$
\begin{aligned}
m\left(r, h_{1}(z)\right) & \sim 2 r /(\log r)^{2}, \\
N\left(r, 1 / h_{1}(z)\right) & \sim 2 r /(\log r)^{2} .
\end{aligned}
$$

Put

$$
H(z)=h(z) h_{1}(z) .
$$

Then $H(z)$ is an entire function (canonical product) of order one, genus zero, and

$$
\log M(r, H)=\log h(r)+\log h_{1}(r) \quad(r>0) .
$$

Since $h(r)>1, h_{1}(r)>1$ for $r>0$, we have by (10)

$$
\int_{1}^{\infty} r^{-2} \log M(r, H) d r=\infty .
$$

Similarly 


$$
\log M(r, H) \geqq \log h_{1}(r) \geqq m\left(r, h_{1}\right) \sim 2 r /(\log r)^{2},
$$

and so the lower order of $H(z)$ is one. Further

$$
N(r, 1 / H) \geqq N\left(r, 1 / h_{1}\right) \sim 2 r /(\log r)^{2} ;
$$

and since the lower order of $h(z)$ is less than $1 / 2$, we have, for a sequence of values of $r$ tending to infinity,

$$
m(r, H) \leqq m\left(r, h_{1}\right)+o\left(r^{1 / 2}\right) .
$$

Combining (18), (19) and (14) we have

$$
\limsup _{r \rightarrow \infty} N(r, 1 / H) / T(r, H)=1, \quad \delta(0, H)=0 .
$$

Applying to $H(z)$ (instead of $h(z))$ the argument in $\S 4$, we see that a suitable primitive $f(z)$ of $H(z)$ has no finite deficient values although its genus is lowered by differentiation. On the other hand, it is clear by (17) and the asymptotic equivalence of $\log M(r, f)$ and $\log M\left(r, f^{\prime}\right)$, that the function $f(z)$ is of regular growth, order one.

\section{REFERENCES}

1. R. Nevanlinna, Le théorème de Picard-Borel et la théorie des fonctions méromorphes, Paris, 1929.

2. G. Valiron, Lectures on the general theory of integral functions, Toulouse, 1923.

3. H. Wittich, Neuere Untersuchungen über eindeutige analytische Funktionen, Berlin, 1955.

SYRACUSE UNIVERSITY AND

Mathematics Research Center, University of Wisconsin 